\title{
Infraestructura portuaria y crecimiento económico regional en México
}

\section{Port infrastructure and regional economic growth in Mexico}

\author{
Isidro ENRique ZePEDA-OrtegA* \\ Gerardo Ángeles-Castro* \\ David Guillermo Carrillo-Murillo**
}

\begin{abstract}
Previous studies about the link between transport infrastructure and regional economic growth have differing conclusions. This paper addresses the problem from the port infrastructure and regional economic growth in Mexico. An index of infrastructure, interconnectivity and port hinterland is determined regionally and by analyzing panel data, the positive influence ports-growth between 1996 and 2013 for all coastal regions is explained. We show that as the level of port infrastructure is increased above a certain level, the marginal effects begin to decline its local impact.
\end{abstract}

Keywords: port infrastructure, transportation, regional economic growth, hinterland.

\section{Resumen}

Estudios previos sobre la relación entre infraestructura de transporte y crecimiento económico regional presentan conclusiones divergentes. Este documento aborda el problema a partir de la infraestructura portuaria regional en México. Se determinó regionalmente un Índice de infraestructura, el hinterland y la interconectividad portuaria, y mediante un análisis de panel de datos, se explica la relación positiva puertos-crecimiento entre 1996 y 2013 para las regiones litorales. Se demuestra que a medida que se incrementa la infraestructura portuaria de una región los efectos marginales empiezan a declinar su impacto local.

Palabras clave: infraestructura portuaria, transporte, crecimiento económico regional, hinterland.

* Instituto Politécnico Nacional, correo-e: ing.isidroenrique@hotmail.com, gangeles@ipn.mx

** DTP Consultores, correo-e: dkdoctorat@hotmail.com 


\section{Introducción}

En México a partir de 1993 inició la administración de los puertos con participación de capital privado con el objetivo de mejorar su organización, eficiencia y eficacia, esto los convierte en áreas de oportunidad para la atracción y aplicación de inversiones en infraestructura como terminales e instalaciones logísticas, así como en negocios portuarios, comerciales e industriales (Paredes, 2007).

Los puertos son de suma importancia ya que se constituyen como puntos de enlace para el flujo de mercancías. A través de los puertos mexicanos se transporta alrededor de un tercio del total de la carga del país, además, durante el periodo 1996-2013, la importación y exportación de mercancías por vía marítima aumentó un promedio anual de 29\% y 1.8\%, respectivamente (Inegi, 2014). Este contraste también se observa en el crecimiento del país y, particularmente, de algunas regiones. En 2013, el PIB per cápita de las regiones más rezagadas representó alrededor de $7 \%$ del PIB per cápita más alto. Esto obliga a buscar la explicación de estas discrepancias regionales y su relación con la dotación de infraestructura.

Aunque existe una amplia literatura, todavía hay poco consenso sobre los efectos exactos de la inversión en infraestructura de transporte y el desarrollo regional (Chandra y Thompson, 2000 y Deng et al., 2014). El debate sigue siendo polémico, los estudios previos han dado diferentes resultados debido a las fuentes de los datos, la desagregación y la especificación del modelo. En la mayoría de los casos, los resultados demuestran un impacto positivo y significativo, pero en otros estudios se concluye que es insignificante o significativamente negativo.

Esta controversia se amplía ya que existe consenso de que para desarrollar una economía la inversión en infraestructura es una condición necesaria, pero la conclusión de los impactos positivos de la misma ha sido debatida por el problema de la causalidad inversa (IFMO, 2007). Por otra parte, en el plano regional: cuando la infraestructura de transporte es muy pequeña, su mejora sirve para construir una red básica de vínculos; pero en regiones bien dotadas, la necesidad de una nueva inversión y su posible impacto son cuestionadas (IFMO, 2007). Esto nos conduce a preguntarnos si la infraestructura de transporte puede tener un grado de eficiencia en su impacto.

Este estudio aporta a este debate, se enfoca particularmente en el efecto de la infraestructura portuaria en México, en contraste con los estudios efectuados sobre China (Zhang, 2008, Hong et al., 2011 y Song y Geenhuizen, 2014) que demuestran que las regiones portuarias presentan mayor crecimiento que las regiones interiores; en México, la situación es inversa, lo que hace peculiar este estudio. Además se incluye la 
consideración del hinterland portuario, bajo el argumento de que la infraestructura de los puertos beneficia no sólo a una región, sino puede dispersarse hacia otras. Se integra en el análisis una propiedad de las redes de transporte (el origen-destino) para posteriormente incluirla dentro de la función de producción, lo que permite, según la teoría neoclásica, explicar la relación existente entre la inversión en infraestructura portuaria y el crecimiento económico en el plano regional mexicano.

La metodología se basa en un panel de datos considerando los beneficios que éste brinda en el análisis de los sistemas de transporte (Paaswell, 1997). Este análisis abarca 17 entidades federativas con litoral en México durante el periodo 1996-2013, debido a que se ha considerado que entre 1993 y 1996, la operación portuaria sufrió cambios en su modelo de administración, lo que permite construir un panel más homogéneo. Se analizan modelos econométricos de efectos fijos y efectos aleatorios en tiempo y en grupo: esta metodología permite explicar el impacto en el crecimiento regional vinculado con la inversión en infraestructura portuaria a través del stock físico histórico, el cual se define mediante el Índice de Infraestructura Portuaria. Finalmente, se identifican las regiones más rezagadas de México y se determina que la infraestructura portuaria de estas regiones constituye uno de los factores que limita su crecimiento y explica el rezago regional existente.

El estudio está organizado de la siguiente forma: en la sección dos se realiza una revisión de la literatura y se presenta la situación actual de la infraestructura portuaria en México y el crecimiento económico del país. En la sección tres se explican la metodología a emplear, la fuente de datos y la construcción del panel de datos. Se revisan varios modelos econométricos que permiten observar la dimensionalidad del conjunto de datos y la relación de largo plazo de las variables.

Los resultados obtenidos después de analizar los efectos fijos y los aleatorios, tanto temporales como regionales de los datos y sus pruebas de cointegración se reportan en la sección cuatro. Asimismo, los resultados de la regresión cuadrática sobre los efectos de los diferentes valores de infraestructura portuaria y el crecimiento regional. Se presentan las conclusiones en las que se demuestra la relación positiva de las variables y se realizan recomendaciones que se derivan de estos resultados.

\section{Revisión de la literatura}

El papel de la infraestructura como motor del crecimiento económico puede explicarse teóricamente al considerarla como un stock de capital. Éste se puede incorporar como una entrada de la función de producción y de esta manera determinar la relación entre la infraestructura y el cre- 
cimiento económico (Barro, 1988). Los estudios previos han mostrado que existen efectos directos e indirectos en esta relación (Aschauer, 1998, Paredes, 2007) y que existe una influencia positiva entre ambas variables (Berechman et al., 2006; Lall, 2007; Singh y Bhanumurthy, 2014). En el caso particular de la infraestructura de transporte, ha sido considerada como "fundamental" (Aschauer, 1989) para fomentar el crecimiento económico, y diversas investigaciones han encontrado efectos positivos significativos (Ashauer, 1998; Moomaw y Williams, 1991; Garcia-Milà y McGuire, 1992; Fujita et al., 2001; Rietveld y Nijkamp, 1992; Démurger, 2001; Cantos et al., 2005; Ozbay et al., 2007; y Hong et al., 2011); sin embargo, sus resultados presentan variaciones. Por ejemplo, Ashauer (1998) encontró que entre la infraestructura y el crecimiento existía una elasticidad de 0,39 a 0,56, los resultados de Garcia-Milà y McGuire presentan elasticidad de 0,04 (Garcia-Milà y McGuire, 1992), mientras que algunos otros (Evans y Karras, 1994; Chandra y Thompson, 2000) encontraron poca evidencia para apoyar algún valor e incluso una hipótesis sobre esta relación.

El análisis mediante la función de producción, aplicando el concepto de accesibilidad (Johansson, 1993), demostró que ésta es un beneficio del transporte, lo que es posible determinar a través de la interacción espacial (Lakshmanan et al., 2001). Este último concepto permitió comprobar que el beneficio es una derrama de efectos espaciales y temporales (Berechman et al., 2006). En esta línea, se intentó explicar la relación a través de un modelo espacial cuadrado y se encontró que la densidad de las carreteras tenía efectos de derrama u onda regional en otros factores como los precios y la actividad económica, aunque no fue posible determinar su participación a través del tiempo (Wu y Gopinath, 2008).

Desde la perspectiva regional, la influencia del transporte en la productividad local también ha sido estudiada. Recientes investigaciones encontraron que la magnitud de la aportación del transporte en el crecimiento depende de la cantidad y calidad de la infraestructura existente: para el caso de China, cuando la densidad de carreteras es menor que 0,17 $\mathrm{km} / \mathrm{km}^{2}$, la relación es insignificante, para la densidad entre 0,17 y 0,38 $\mathrm{km} / \mathrm{km}^{2}$ el impacto positivo fue de 0,23 , pero al superar la última densidad, el impacto se redujo a 0,09. En una primera provisión básica de infraestructura de transporte ésta no constituye una red, y por tanto no actúa como motor de la economía local, por otro lado, por encima del valor superior, los efectos positivos tienden a reducirse significativamente (Deng et al., 2014).

Entre los estudios relativos a los puertos se encontró evidencia de su impacto sobre el crecimiento económico regional de las provincias portuarias de China (Hong et al., 2011 y Song y Geenhuizen, 2014), me- 
diante la aplicación de un modelo de regresión lineal y un panel de datos se encontraron elasticidades de 0,13 a 0,19 y de 0,54 a 0,81 , respectivamente. El análisis mostró que la distribución de la infraestructura de transporte espacialmente es una causa importante de las distorsiones económicas entre las regiones chinas. Asimismo, demostró que mayor infraestructura de carreteras incrementa el impacto regional de los puertos debido a su accesibilidad.

Respecto de la metodología a emplear en el análisis empírico para determinar las relaciones entre inversión, infraestructura y crecimiento económico, se han usado las series de tiempo y los cortes transversales (Yoo, 2006). Para la evaluación de los impactos de los sistemas de transporte, los paneles han sido más frecuentemente utilizados debido a que pueden proveer información de cómo los miembros del panel o subconjuntos de ellos, responden o han respondido a cambios en la variable y cómo pesan los factores que influyen en su respuesta (Paaswell, 1997). Una de las ventajas de los paneles es la posibilidad de capturar el cambio de comportamiento en el tiempo, así como medir los componentes de cambios individuales y los agregados, lo cual quedó demostrado después de analizar 60 estudios empíricos en los que se emplearon paneles de datos en diferentes disciplinas (Raimond y Hensher, 1997).

En cuanto al análisis en el plano regional, los paneles de datos han sido empleados para examinar el papel de la infraestructura en el crecimiento ya que permite un análisis tanto temporal como espacial, con resultados satisfactorios para estudios sobre China (Mody y Wang, 1997; Démurger, 2001; Zhang, 2008; Hong et al., 2011; Deng et al., 2014) e India (Singh y Bhanumurthy, 2014).

En el caso particular de México, el estudio del impacto de la inversión pública en infraestructura sobre el crecimiento, empleando un modelo neoclásico, demostró que las inversiones son condiciones insuficientes, ya que se debe complementar con políticas que incrementen el financiamiento, el uso de la infraestructura, así como la eficiencia en la aplicación de los recursos públicos (Aschauer, 1998). Particularmente la infraestructura de transporte resultó estar más relacionada con el crecimiento en las regiones económicas intermedias y no así en las pobres (Looney y Frederiksen, 1981). Al estudiar la disparidad del sureste mexicano, se demostró que el acceso a mercados a través de mejoras en la infraestructura de transporte que vincula áreas urbanas benefician la productividad regional (Deichmann et al., 2004); sin embargo, el estudio de la infraestructura portuaria mexicana en el plano regional continua abierto. 


\subsection{Infraestructura portuaria en México}

En 1993, se inició una etapa de restructuración del sistema portuario mexicano con la integración de capital privado en la inversión física y en la administración de los puertos. Esta inversión ha generado la expansión de la infraestructura portuaria hasta llegar a las condiciones actuales.

En 2013, el sistema portuario mexicano estaba conformado por 102 puertos y 15 terminales fuera de puerto, 58 en el Pacífico y 59 en el Golfo de México y el Caribe; 69 eran para tráfico de altura y cabotaje y 48 únicamente de cabotaje como se aprecia en el mapa 1. De los 102 puertos, 64 funcionan bajo un régimen de concesión: 31 puertos entregados a 16 sociedades mercantiles (Administración Portuaria Integral) para el uso, aprovechamiento y explotación de los bienes y la prestación de los servicios respectivos, tres a entidades paraestatales turísticas, 24 a gobiernos estatales, cinco a gobiernos municipales y un puerto privado. La federación mantiene la administración de 38 puertos (SCT, 2013).

El sistema portuario movilizó 288 millones de toneladas de carga total en 2013. En la década de 1996 a 2006 presentó un incremento de $31 \%$ (3.3\% anual), mientras que entre 2007 y 2013 aumentó 5.7\% (0.9\% anual), pese al retroceso sufrido en 2009. La participación de cada uno de los 102 puertos localizados en 17 entidades federativas, se observa en la tabla 1.

En 2006, la carga comercial representó 43\% y la petrolera 57\%, mientras que en 2013, 58\% y 42\%, respectivamente. En el periodo de 2007 a 2013 el volumen transportado mediante cabotaje (trafico nacional corto) se redujo en $3.8 \%$, (-0.63\% anual) para participar del total de carga en $25.2 \%$, mientras que el volumen transportado en viaje de altura se incrementó $1.95 \%$ (0.32\% anual) para lograr una participación de $74.8 \%$. El volumen de altura se repartió $63 \%$ para los puertos del golfo y $37 \%$ para los del pacífico, mientras el cabotaje se repartió $75 \%$ y $25 \%$ a través del golfo y pacífico, respectivamente.

Existen diferencias en el aprovechamiento y operación de los distintos puertos. En 2013, sólo nueve puertos concentraron 84\% del total de carga: en el Golfo de México, el puerto de Cayo de Arcas 22.2\% (Campeche), Veracruz 9.5\% (Veracruz), Coatzacoalcos 10.1\% (Veracruz), Altamira 7.1\% (Tamaulipas), Tuxpan 5.3\% (Veracruz) y Punta Venado 3.8\% (Quintana Roo), en tanto que en el pacífico, Lázaro Cárdenas 11.6\% (Michoacán), Manzanillo 11.1\% (Colima) e Isla Cedros 3.5\% (Baja California Sur).

La carga contenerizada aumentó $59.2 \%$ en el periodo 2007-2013 (9.9\% anual). En 2013 representó $13.8 \%$ del total de carga movida a través de los puertos mexicanos (incluyendo petróleo) concentrándose en 


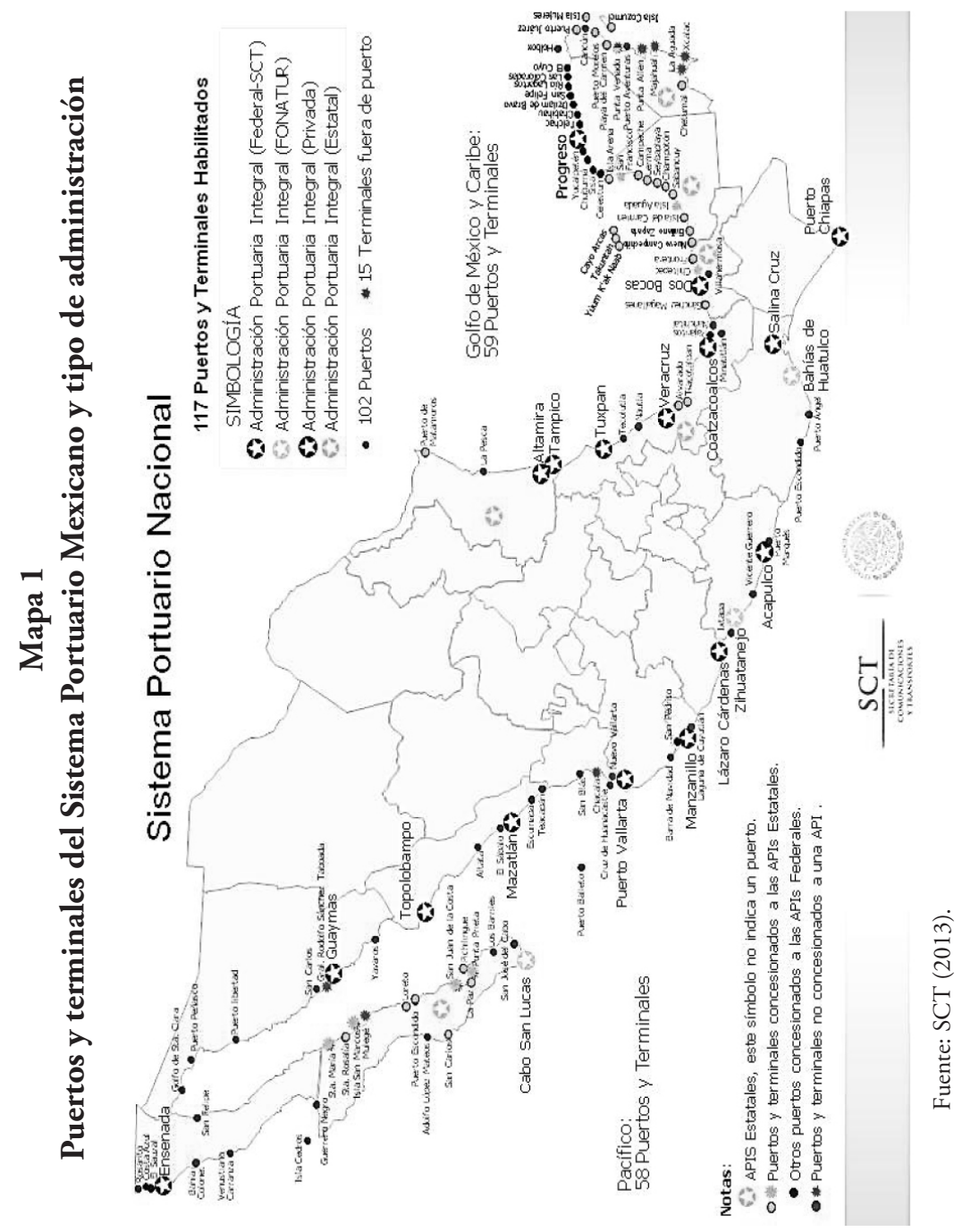




\section{Tabla 1}

Puertos mexicanos de acuerdo con su ubicación (litoral y entidad federativa), la composición de su tráfico comercial (internacional o nacional) y su participación en la carga general

\begin{tabular}{|c|c|c|c|c|c|c|}
\hline \multirow{2}{*}{$\begin{array}{l}\text { Litoral Entidad } \\
\text { federativa }\end{array}$} & \multirow[t]{2}{*}{ Total } & \multirow[t]{2}{*}{ Puertos } & \multirow[t]{2}{*}{ Terminales } & \multicolumn{2}{|c|}{ Tipo de tráfico } & \multirow{2}{*}{$\begin{array}{c}\text { Participación } \\
\text { de carga } \\
\text { (porcentaje) }\end{array}$} \\
\hline & & & & Altura & Cabotaje & \\
\hline TOTAL NACIONAL & 117 & 102 & 15 & 69 & 117 & 100 \\
\hline Pacífico & 58 & 51 & 7 & 38 & 58 & 45.6 \\
\hline Baja California & 8 & 8 & 0 & 6 & 8 & 5.16 \\
\hline Baja California Sur & 16 & 11 & 5 & 12 & 16 & 1.22 \\
\hline Colima & 3 & 3 & 0 & 3 & 3 & 12.47 \\
\hline Chiapas & 1 & 1 & 0 & 1 & 1 & 0.03 \\
\hline Guerrero & 5 & 5 & 0 & 5 & 5 & 0.05 \\
\hline Jalisco & 2 & 2 & 0 & 2 & 2 & - \\
\hline Michoacán de Ocampo & 1 & 1 & 0 & 1 & 1 & 11.60 \\
\hline Nayarit & 5 & 4 & 1 & 1 & 5 & - \\
\hline Oaxaca & 4 & 4 & 0 & 2 & 4 & 1.87 \\
\hline Sinaloa & 6 & 6 & 0 & 3 & 6 & 1.51 \\
\hline Sonora & 7 & 6 & 1 & 2 & 7 & 1.86 \\
\hline $\begin{array}{l}\text { Golfo de México y Mar } \\
\text { Caribe }\end{array}$ & 59 & 51 & 8 & 31 & 59 & 54.4 \\
\hline Campeche & 14 & 12 & 2 & 8 & 14 & 22.23 \\
\hline Quintana Roo & 14 & 9 & 5 & 10 & 14 & 3.79 \\
\hline Tabasco & 5 & 4 & 1 & 2 & 5 & 3.43 \\
\hline Tamaulipas & 4 & 4 & 0 & 3 & 4 & 8.91 \\
\hline Veracruz & 10 & 10 & 0 & 5 & 10 & 24.91 \\
\hline Yucatán & 12 & 12 & 0 & 3 & 12 & 0.97 \\
\hline
\end{tabular}

Fuente: elaboración propia con datos de Inegi (2013) y SCT (2013).

el puerto de Manzanillo con más del doble de contenedores desplazados respecto a sus competidores Lázaro Cárdenas y Veracruz. En cuanto al tráfico de buques y transbordadores los puertos del estado de Quintana Roo concentran $22.9 \%$. En el arribo de cruceros, $89.9 \%$ de operaciones y movimiento de pasajeros se concentran en Quintana Roo (68.7\%), Baja California Sur (16.1\%) y Yucatán (5.1\%).

\subsection{Crecimiento económico y regiones}

El Inegi (2010) presentó una división geográfica del territorio mexicano en siete regiones considerando sus similitudes en características históricas, sociales, culturales y socioeconómicas, que sin embargo poco aporta para comprender los cambios que persigue este estudio. La discusión acerca 
de como considerar una región portuaria es amplia (Ducruet, 2009), su cálculo es complejo ya que requiere tomar en cuenta, por un lado, una extensión geográfica y, por el otro, las relaciones entre la región y el puerto tanto positivas como negativas (Paredes, 2007) y las relaciones sistémicas del puerto con otros puertos en la misma región.

Inicialmente, para delimitar la extensión geográfica se utilizarán como regiones de estudio a los estados con litoral que disponen de infraestructura portuaria, considerando que los registros del crecimiento económico en México (PIB) se encuentran agregados por Estados, acotando así las regiones de estudio y su Producto Interno Bruto que se muestran en la tabla 2.

Se puede observar que el sector terciario representa en general más de $50 \%$ del PIB, excepto para Campeche, el cual presenta una composición atípica debido a la actividad petrolera que es afectada por la volatilidad de los precios del petróleo, razón por la cual, se eliminan del presente análisis los puertos de dicha región (Inegi, 2013).

\section{Metodología}

\subsection{Especificación del modelo}

Los estudios anteriores (Garcia-Milà et al., 1996; Aschauer, 1998; Song y Geenhuizen, 2014) han empleado una función de producción tipo Cobb-Douglas donde la inversión en infraestructura es interpretada como un capital privado para el cual se asumen retornos de inversión positivos. Se empleó esta función para probar la infraestructura de transporte marítimo como una entrada de la función de producción y por su facilidad de comparación con otros estudios.

$$
Y_{i t}=A f\left(K_{i t}, L_{i t}\right)=A K_{i t}^{\alpha} L_{i t}^{\beta}
$$

Donde $Y_{i t}$ es la producción de la región $i$ en el momento $t, A$ una mejora tecnológica que afecta el total de la función, $K_{i t}$ es el stock de capital y $L_{i t}$ la masa laboral respectivos. En cuanto a la masa laboral $\left(\mathrm{L}_{\mathrm{it}}\right)$, se utilizó la población económicamente activa de la región $i$ en el periodo t, $\left(\right.$ PEA $A_{i t}$.

Para incorporar en esta función el stock de capital, se debe considerar la problemática existente en México respecto a la ineficiencia en la aplicación del capital destinado a la inversión en infraestructura (Aschauer, 1998). En su lugar, se utilizará una variable proxy que permitirá identificar cuantitativamente los elementos de inversión física en infraestructura portuaria existente y compararlos a lo largo del tiempo, que incluirá las mejoras tecnológicas y evitará afectar el cálculo por la ineficiencia en 


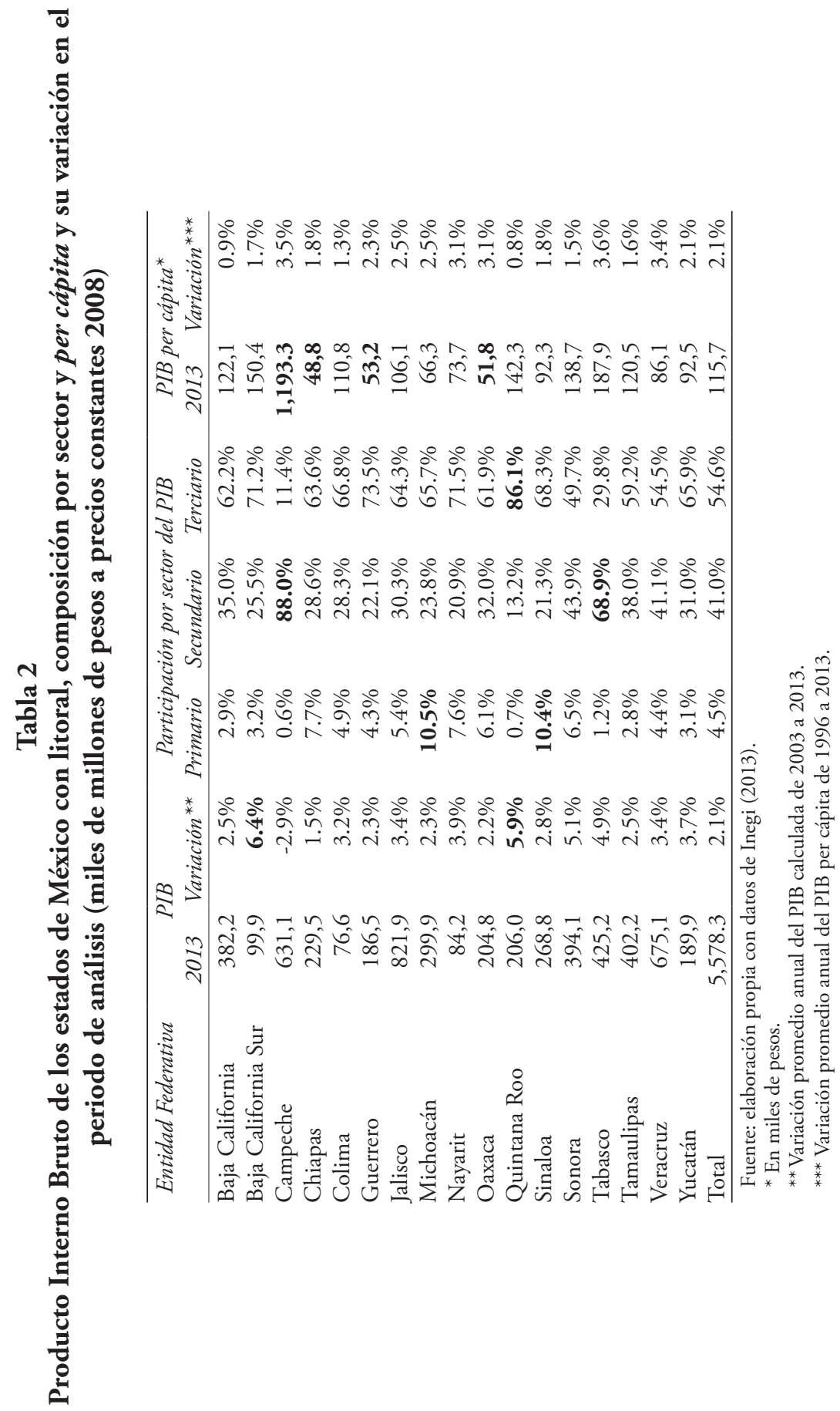


la aplicación del gasto, la cual está presente en los países en desarrollo (Aschauer, 1998) (Ahmed et al., 2013). La variable se ha denominado Factor de Infraestructura Portuaria FIP, y está definida como:

$$
F I P_{t i}=\frac{\sum_{1}^{n} A_{t i}}{\bar{A}_{\iota}}+\frac{\sum_{1}^{n} C_{t i}}{\bar{C}_{\iota}}+\frac{\sum_{1}^{n} P_{t i}}{\bar{P}_{\iota}}+\frac{\sum_{1}^{n} O_{t i}}{\bar{O}_{\iota}}+\frac{\sum_{1}^{n} D_{t i}}{\bar{D}_{\iota}}
$$

Donde: $i$ es la región de análisis

$t$ es el año dentro del periodo de análisis

$n$ es el número de puertos localizados en la región $i$

$A$ es la longitud de atraque de los muelles con capacidad internacional disponibles

$C$ es la longitud de atraque de los muelles empleados para transporte local (cabotaje)

$P$ es la longitud de atraque de los muelles de actividades pesqueras disponibles

$T$ es la suma de las longitudes de atraque para actividades turísticas y arribo de cruceros

O es la longitud de atraque de uso relacionado con actividades petroleras disponibles

$\mathrm{D}$ es la longitud de atraque de usos diversos disponibles

Otro aspecto que merece atención es el hinterland del puerto, entendido como la zona del mercado de un puerto (Carrillo, 2011) esto es, la zona de influencia de los bienes que pasan a través de un puerto hasta llegar a su destino final. El hinterland del sistema portuario permite delimitar la región portuaria (Ducruet, 2009) y se puede analizar a través de los elementos que lo describen: los modos de transporte, los componentes de red (nodos y enlaces) y actores de oferta y demanda (Carrillo, 2011).

En cuanto al elemento del hinterland correspondiente a los modos de transporte, se ha incorporado en el modelo una variable proxy de conexión intermodal de la región mediante el cálculo de la densidad de infraestructura carretera Dencar. Esto resulta relevante si consideramos que los principales puertos han expandido su área de influencia, entre otros aspectos, debido a su integración con otros modos (lo que para otros puertos puede convertirse en una limitante si el puerto no tiene interconexiones modales de transporte). Estudios recientes han empleado variables proxy para medir cuantitativa y cualitativamente las características de diversos modos de transporte y comparar su impacto en el crecimiento de forma exitosa (Hong et al., 2011 y Song y Geenhuizen, 2014) por lo que siguiendo estos modelos podemos definir para las regiones de México la densidad de carreteras como: 


\section{DenCar = longitud de carreteras/ área geográfica}

En cuanto a la oferta y demanda, como una segunda característica del hinterland del puerto (Carrillo 2011), en este estudio se ha propuesto utilizar una variable proxy en forma de coeficiente, el cual separa los efectos de derrama (spillover) económica del puerto sobre otras regiones, del impacto en la propia región donde se ubica el puerto y el cual se ha denominado Coeficiente de regionalización propia del hinterland por su mercado $H_{M K}$ Se calculó a partir de la construcción de una matriz origendestino de las mercancías que pasan por cada puerto localizado en cada una de las regiones. Debido a la ausencia de datos históricos, se calculó con valores del 2013 y se consideró estático para todo el periodo ${ }^{1}$, de la siguiente forma:

$$
H M K_{i}=\frac{\sum_{n=1}^{N} O_{n i} D_{i}}{\sum_{n=1}^{N} O_{n i} D_{j}}
$$

Donde: $O_{n} D_{i}$ es la carga con origen en el puerto n de la región i con destino en la región i

$O D_{j}$ es la carga con origen en el puerto n de la región i con destino en cualquier región

El coeficiente $H_{M K}$ se aplicó para corregir el FIP de cada región como un efecto de concentración sobre la misma región donde se ubica el puerto debido al beneficio obtenido al atender la demanda de uso de la infraestructura: FIPred $=H_{M K} \times$ FIP.

La conectividad, además de referirse a los flujos de mercancía, se refiere al flujo internacional de personas. Siguiendo el criterio de Song y Geenhuizen (2014), los efectos de la infraestructura aeroportuaria disponible en la región se integraron al modelo con una variable referente al número de salidas de vuelos internacionales comerciales regulares realizados en los aeropuertos de la región (SAL) (Song y Geenhuizen, 2014). La función de producción de la región $i$ en el momento $t$, está definida como:

$$
Y_{t i}=f\left(\text { FIPred }_{t i} \text { Dencar, } \text { Sal }_{t i}, P E A_{t i}\right)
$$

\footnotetext{
${ }^{1}$ Constituye una limitación importante de este estudio, ya que no permite observar los efectos temporales del hinterland y la infraestructura, pero permite obtener un primer acercamiento sobre el comportamiento de las variables en estudio. Esto abre una posibilidad para una investigación posterior más amplia.
} 
Los parámetros de estimación pueden ser obtenidos mediante la linealización de la función de producción en términos logarítmicos, y así determinar la elasticidad de cada regresor de la siguiente forma:

$$
\left.\operatorname{LnPIB}_{t i}=\beta_{0}+\beta_{1} \text { LnFIPred }_{t i}+\beta_{2} \text { LnDencar }_{t i}+\beta_{3} \operatorname{Ln}_{\text {Sal }}+\beta_{t i} \operatorname{LnPEA}_{t i}\right)
$$

\subsection{Datos}

Se tabuló un panel de datos con los indicadores de 17 regiones portuarias de México y registros temporales de 1996 a 2013. El PIB regional (Y), presentado en la tabla 2, fue calculado a precios constantes de 2008 a partir de los datos publicados por el Inegi (2014). En la tabla 3 se presentan los valores adimensionales del Factor de Infraestructura Portuaria (FIPred) y del Coeficiente de regionalización propia del hinterland $\left(H_{M K}\right)$ determinados para cada región tras revisar las características de infraestructura disponibles en cada uno de los 102 puertos con la información del Catastro Portuario Nacional (SCT, 2015) y la información geográfica de las regiones proporcionada por el Inegi (2013).

El incremento promedio anual del PIB y del FIB de las regiones de estudio se muestra en el mapa 2 y mapa 3, respectivamente, en donde se observa que algunas regiones han realizado incrementos en su infraestructura mientras que otras, como Veracruz, relativamente no han aumentado significativamente su infraestructura en el periodo 1996 -2013.

En la tabla 4, se muestra la estadística descriptiva de las variables del modelo: PIB (millones de pesos), FIPred (adimensional), Dencar $\left(\mathrm{km} / 10,000 \mathrm{~km}^{2}\right), S A L$ (número de vuelos anuales), PEA (número de personas). La densidad de infraestructura carretera y las salidas internacionales de cada región se obtuvieron de estadísticos del sector transporte elaborados por la SCT (2013), por su parte la población económicamente activa se obtuvo de los registros de Inegi (2013).

Se aplicó a los datos la prueba de raíz unitaria bajo el criterio de LevinLin-Chu (Levin et al., 2002) y la prueba de cointegración tipo Engle Granger para garantizar la fiabilidad de los resultados de la regresión.

Los resultados de la primera muestran que el logaritmo de las variables del modelo no contiene raíces unitarias en niveles con un rezago y son estacionarias, excepto el factor de infraestructura (FIPred) que no es estacionario. En cuanto a la segunda prueba, el panel se encuentra cointegrado, por lo tanto, los datos tienen relación de largo plazo. Los resultados se muestran en la tabla 5 . 
Tabla 3

Factor de Infraestructura Portuaria (FIP) y Coeficiente de regionalización propia del hinterland por su mercado $\left(H_{M K}\right)$ de cada región

\begin{tabular}{lrrrrr}
\hline \multicolumn{1}{c}{ Región } & \multicolumn{2}{c}{ Periodo } & \multicolumn{2}{c}{ Incremento } & $H_{M K}$ \\
& 1996 & 2013 & Total & $\begin{array}{r}\text { Promedio } \\
\text { anual }\end{array}$ \\
\hline Baja California & 4,61 & 5,86 & $27.1 \%$ & $1.6 \%$ & 0,9709 \\
Baja California Sur & 4,63 & 8,91 & $92.4 \%$ & $5.4 \%$ & 0,3271 \\
Campeche & 5,44 & 16,49 & $203.4 \%$ & $11.9 \%$ & 0,9753 \\
Chiapas & 0,96 & 1,14 & $18.4 \%$ & $1.1 \%$ & 0,4397 \\
Colima & 5,93 & 7,28 & $22.8 \%$ & $1.3 \%$ & 0,4420 \\
Guerrero & 2,41 & 4,83 & $101.0 \%$ & $5.9 \%$ & 0,0183 \\
Jalisco & 2,47 & 3,67 & $48.6 \%$ & $2.8 \%$ & 1,0000 \\
Michoacán de Ocampo & 2,19 & 5,93 & $170.8 \%$ & $10.0 \%$ & 0,3018 \\
Nayarit & 0,96 & 1,72 & $79.5 \%$ & $4.7 \%$ & $\mathrm{~N} / \mathrm{D}$ \\
Oaxaca & 2,60 & 2,68 & $3.1 \%$ & $0.2 \%$ & 0,3844 \\
Quintana Roo & 5,87 & 8,20 & $39.7 \%$ & $2.3 \%$ & 0,9926 \\
Sinaloa & 5,89 & 7,27 & $23.5 \%$ & $1.4 \%$ & 0,3633 \\
Sonora & 5,46 & 11,68 & $113.9 \%$ & $6.7 \%$ & 0,5533 \\
Tabasco & 2,14 & 4,85 & $126.0 \%$ & $7.4 \%$ & 0,9687 \\
Tamaulipas & 9,57 & 15,90 & $66.3 \%$ & $3.9 \%$ & 0,5908 \\
Veracruz & 17,41 & 21,27 & $22.2 \%$ & $1.4 \%$ & 0,4961 \\
Yucatán & 3,44 & 6,10 & $77.3 \%$ & $4.5 \%$ & 0,6747 \\
Promedio general & 4,80 & 8,07 & $77.6 \%$ & $4.9 \%$ & \\
\hline
\end{tabular}

Fuente: elaboración propia con datos de SCT (2015).

Tabla 4

Estadística descriptiva de los valores de la región portuaria empleados en el modelo

\begin{tabular}{|c|c|c|c|c|c|c|}
\hline & Variable & Datos & Media & $\begin{array}{l}\text { Desviación } \\
\text { estándar }\end{array}$ & Min & $\operatorname{Max}$ \\
\hline PIB & $\begin{array}{l}\text { Producto Interno } \\
\text { Bruto }\end{array}$ & 288 & 247652,3 & 166929,7 & 42087,51 & 821822,1 \\
\hline FIPred & $\begin{array}{l}\text { Factor de Infraes- } \\
\text { tructura Portuaria }\end{array}$ & 288 & 6,715 & 4,637 & 0,937 & 21,270 \\
\hline DenCar & $\begin{array}{l}\text { Densidad de in- } \\
\text { fraestructura carre- } \\
\text { tera }\end{array}$ & 288 & 0,366 & 0,177 & 0,0707 & 0,840 \\
\hline SAL & $\begin{array}{l}\text { Salidas de vuelos } \\
\text { internacionales }\end{array}$ & 288 & 7497,1 & 13534,6 & 0,0 & 68422,0 \\
\hline PEA & $\begin{array}{l}\text { Población económi- } \\
\text { camente activa }\end{array}$ & 288 & 1241869 & 800684,6 & 167543 & 3548171 \\
\hline
\end{tabular}

Fuente: elaboración propia empleando Stata 13. 


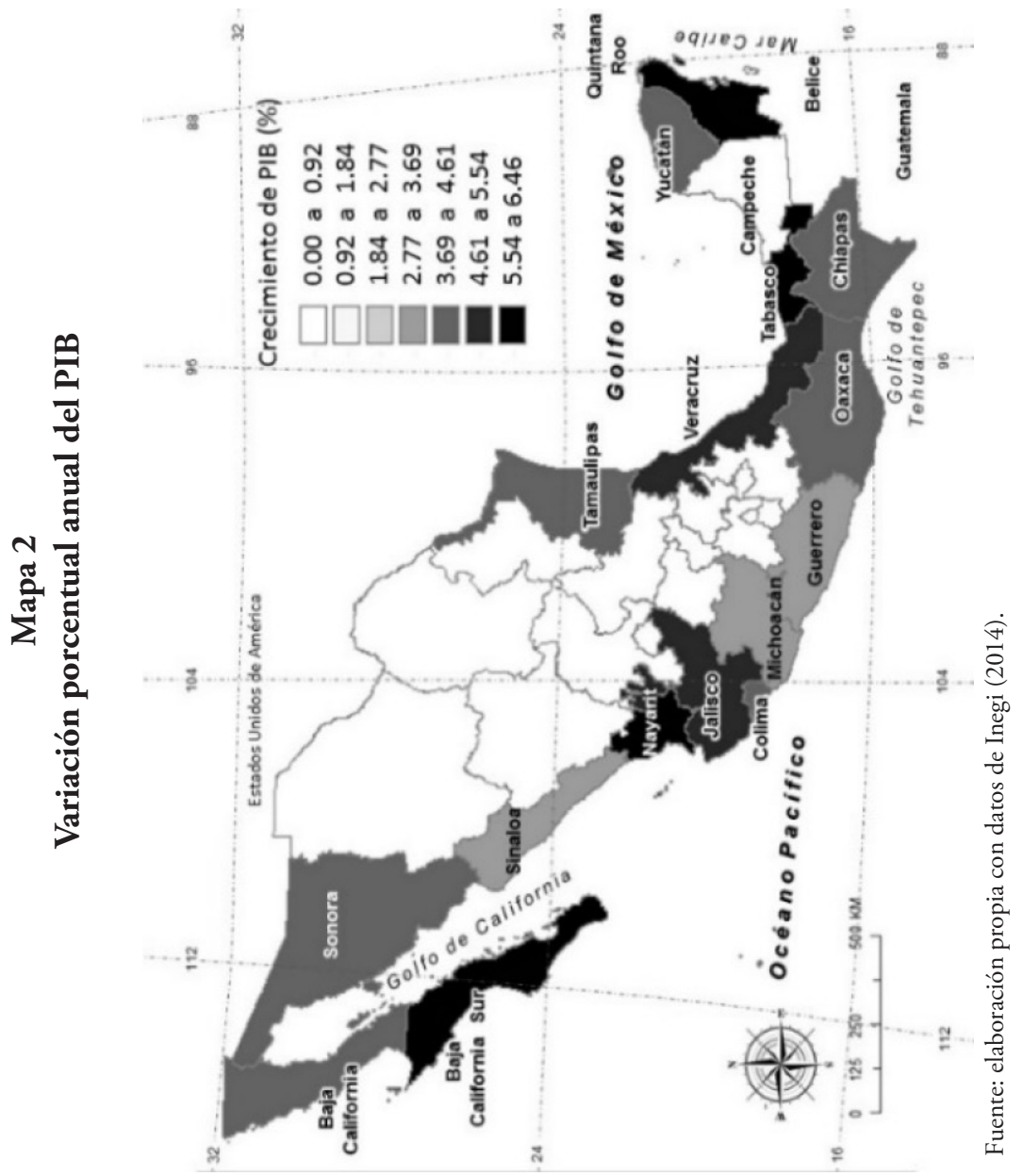




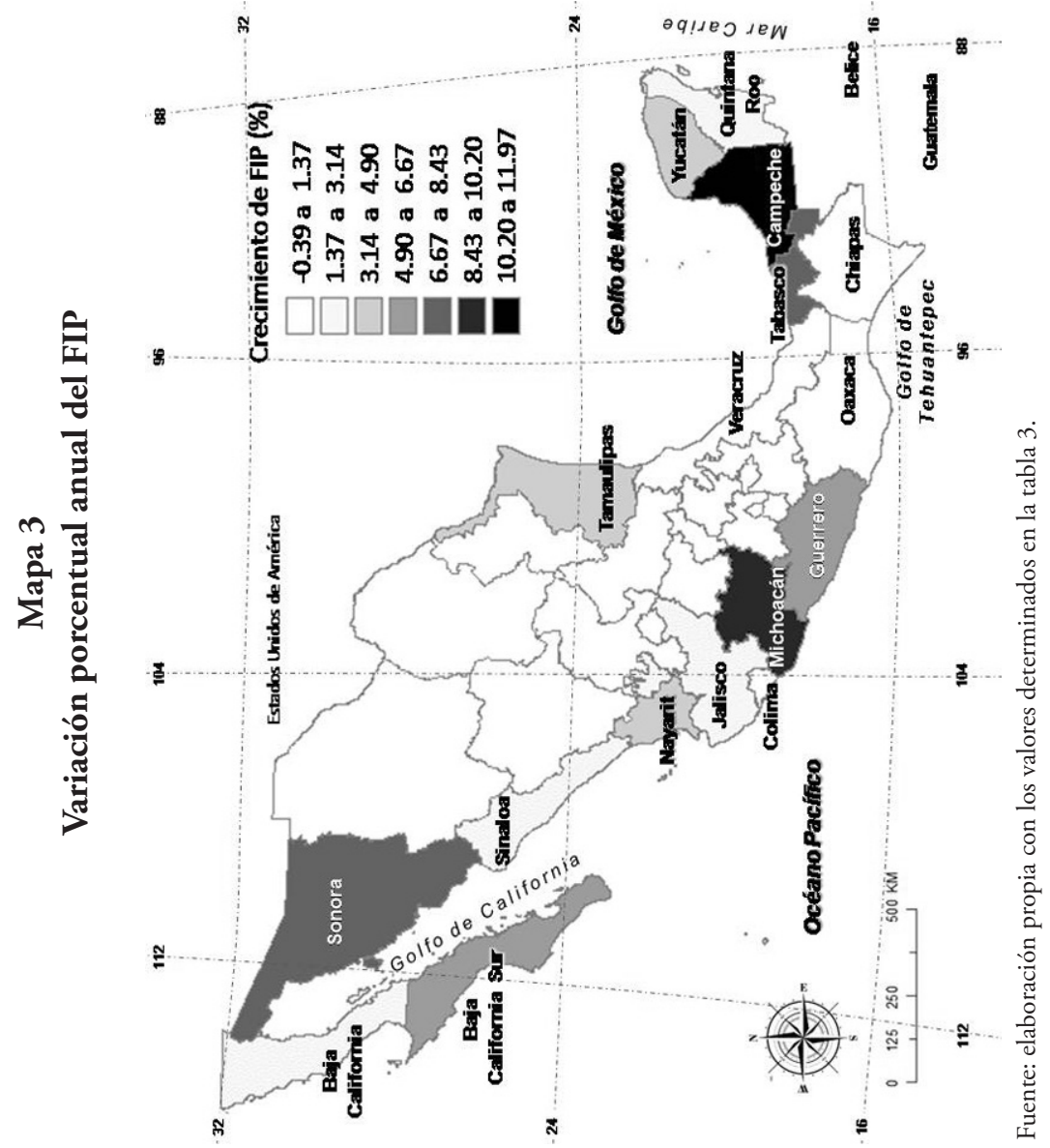


Tabla 5

\section{Resultados de la prueba de raíz unitaria y de la prueba de cointegración}

\begin{tabular}{clccl}
\hline Prueba & Variable & $\begin{array}{c}t^{*} \\
\text { ajustada }\end{array}$ & Valorp & $\begin{array}{c}\text { Especificación y estructura de rezagos } \\
\text { de Dickey-Fuller Aumentada }\end{array}$ \\
\hline Raíz unitaria & Ln PIB & $-5,2845$ & 0,0000 & Tendencia y un rezago \\
& Ln FIPred & 30,6434 & 1,0000 & Tendencia y un rezago \\
& Ln Dencar & $-3,5237$ & 0,0002 & Tendencia y un rezago \\
& Ln Sal & $-3,6577$ & 0,0001 & Tendencia y un rezago \\
& Ln PEA & $-3,2534$ & 0,0006 & Tendencia y un rezago \\
Cointegración & Residual & $-5,0541$ & 0,0000 & (Engle Granger) \\
\hline
\end{tabular}

Fuente: elaboración propia con los resultados de Stata 13.3.

\section{Resultados}

Se realizó un análisis del panel mediante tres modelos: mínimos cuadrados ordinarios, efectos aleatorios y efectos fijos para determinar los coeficientes de las variables, sus resultados se presentan en la tabla 6 .

Se resalta que todas las regiones presentan un intercepto en el PIB positivo y estadísticamente significativo lo que nos refiere a que el modelo es consistente. Además, se realizó un análisis desagregando los coeficientes del modelo para observar los valores de los interceptos para cada una de las regiones estatales, esto mediante un modelo de mínimos cuadrados ordinarios con variables dummies dicótomas (OLSDV por sus siglas en inglés).

Al aplicar la prueba que Breusch y Pagan formularon como Prueba del Multiplicador de Lagrange se encontró que sí existen efectos aleatorios, no obstante, al realizar la prueba de Hausman, se comprobó que el modelo más satisfactorio es el de Efectos fijos. Dentro de los modelos analizados, el modelo de efectos aleatorios de tiempo no presenta resultados consistentes, por lo que no se considera adecuado aplicarlo. Posteriormente, se realizó una regresión de mínimos cuadrados ordinarios empleando variables dummies interactivas para obtener los efectos desagregados por cada región de las variables explicativas sobre la dependiente los cuales se encuentran en la tabla 7.

Los coeficientes desagregados del FIPred obtenidos mediante la regresión (dummies interactivas) de la tabla 7, representan el Impacto Marginal del FIP en el PIB (IMFP) desagregado por región. Con estos, se realizó un análisis de regresión cuadrática a fin de determinar si diferentes condiciones de infraestructura portuaria están relacionados con su impacto 


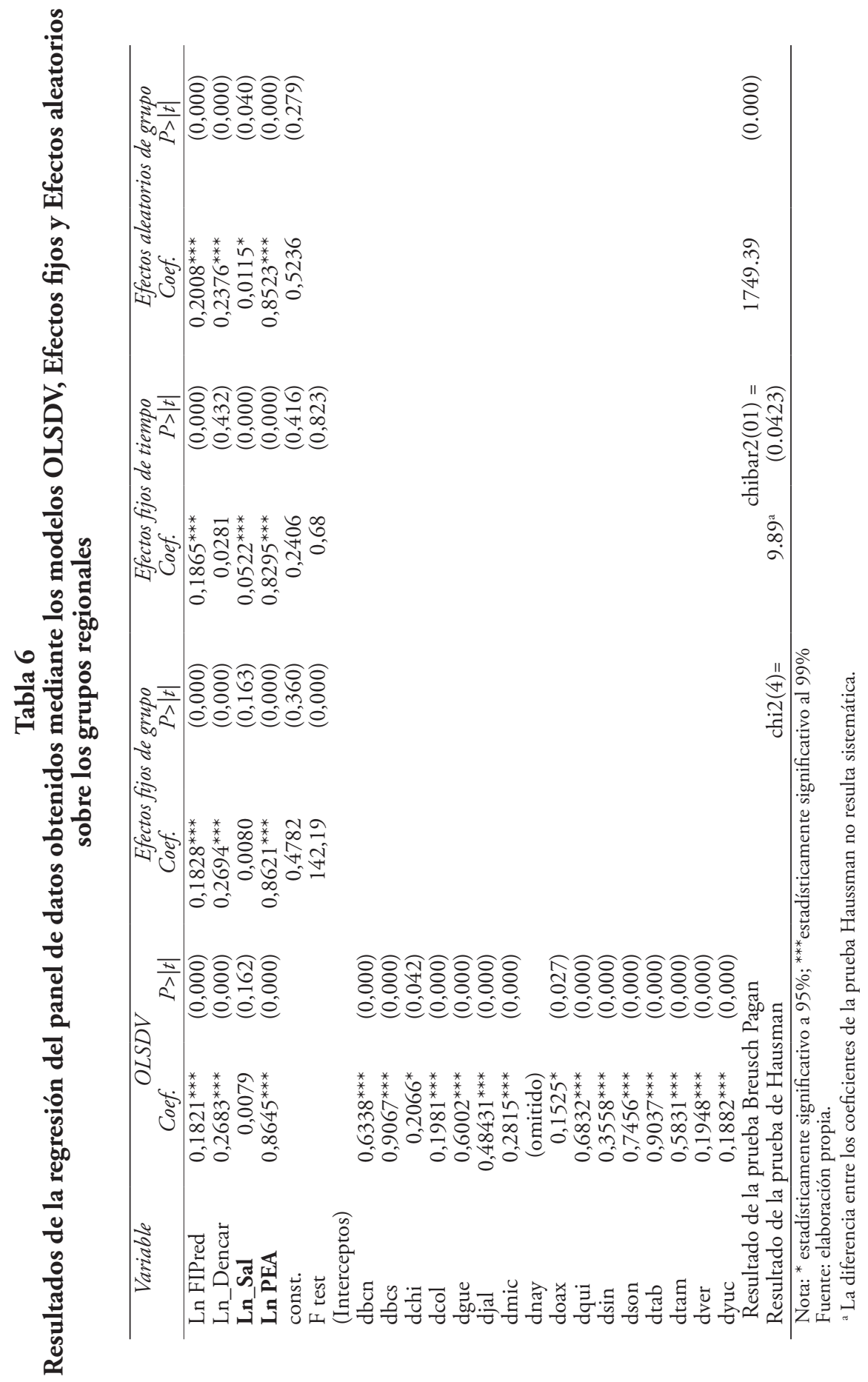


en el PIB mediante el modelo Impacto $($ IMFP $)=\varphi_{1}$ FIPred $_{P R O M}{ }^{2}+\varphi_{2}$ FIPred $_{P R O M}$ Los resultados se presentan en la tabla 8.

La ecuación que define la relación entre el IMFP y el FIP promedio tiene la forma $I M F P=-0,2550 F I P^{2}+0,012$ FIP. Al trazar los resultados en la gráfica 1 , se observa que a medida que el valor de infraestructura portuaria (FIPred) crece, el impacto marginal sobre el PIB también lo hace hasta cierto valor, a partir del cual empieza a decrecer. Los resultados de la regresión son altamente significativos.

\subsection{Discusiones}

Los resultados obtenidos para los tres modelos (tabla 6) son consistentes entre sí: las variables relevantes tienen coeficientes positivos con alta significancia y valores similares entre los modelos.

La infraestructura portuaria disponible ( $L n F I P)$, la red de infraestructura carretera ( $L n D e n c a r)$ y la población económicamente activa ( $L n P E A)$ tienen influencia positiva con el $P I B$ de las regiones mexicanas, y son estadísticamente significativas a $99 \%$, mientras que la interconectividad aérea internacional no resulta estar relacionada con el $P I B$ regional porque no es estadísticamente significativa a niveles convencionales. Esta última conclusión resulta consistente con los resultados obtenidos en otras investigaciones (Hong et al., 2011). Un incremento marginal de uno por ciento en el FIP puede influenciar un aumento de 0.18 a 0.20 por ciento en el $P I B$ de la región, mientras que una adición de uno por ciento en la densidad carretera (DenCar) ceteris paribus podría provocar 0.23 a 0.26 por ciento adicional de PIB. La PEA influye altamente en el PIB regional con valores de coeficientes de la regresión de 0.82 a 0.86 .

La influencia regional de la infraestructura de un puerto es afectada por las condiciones de uso generadas por la demanda de servicio de transporte. Queda mostrado que el hinterland portuario afecta la influencia económica regional local del puerto y sitúa los beneficios de la infraestructura portuaria a otras regiones.

A partir de los interceptos (modelo OLSDV) positivos y altamente significativos (con niveles de significancia de 95 y 99\%) se aprecia que existe una magnitud de actividad económica positiva al inicio del periodo de estudio, lo que permite afirmar que el modelo es consistente: Baja California Sur (BCS) y Tabasco serían las regiones que corresponden a los valores de intercepto mayores. En el caso de Tabasco, esto se puede explicar por su relación con la actividad petrolera, en cuanto a BCS, mientras que su sector primario y secundario aportan poco más de $28 \%$ del PIB del estado, solamente dos actividades del sector terciario: Hote- 


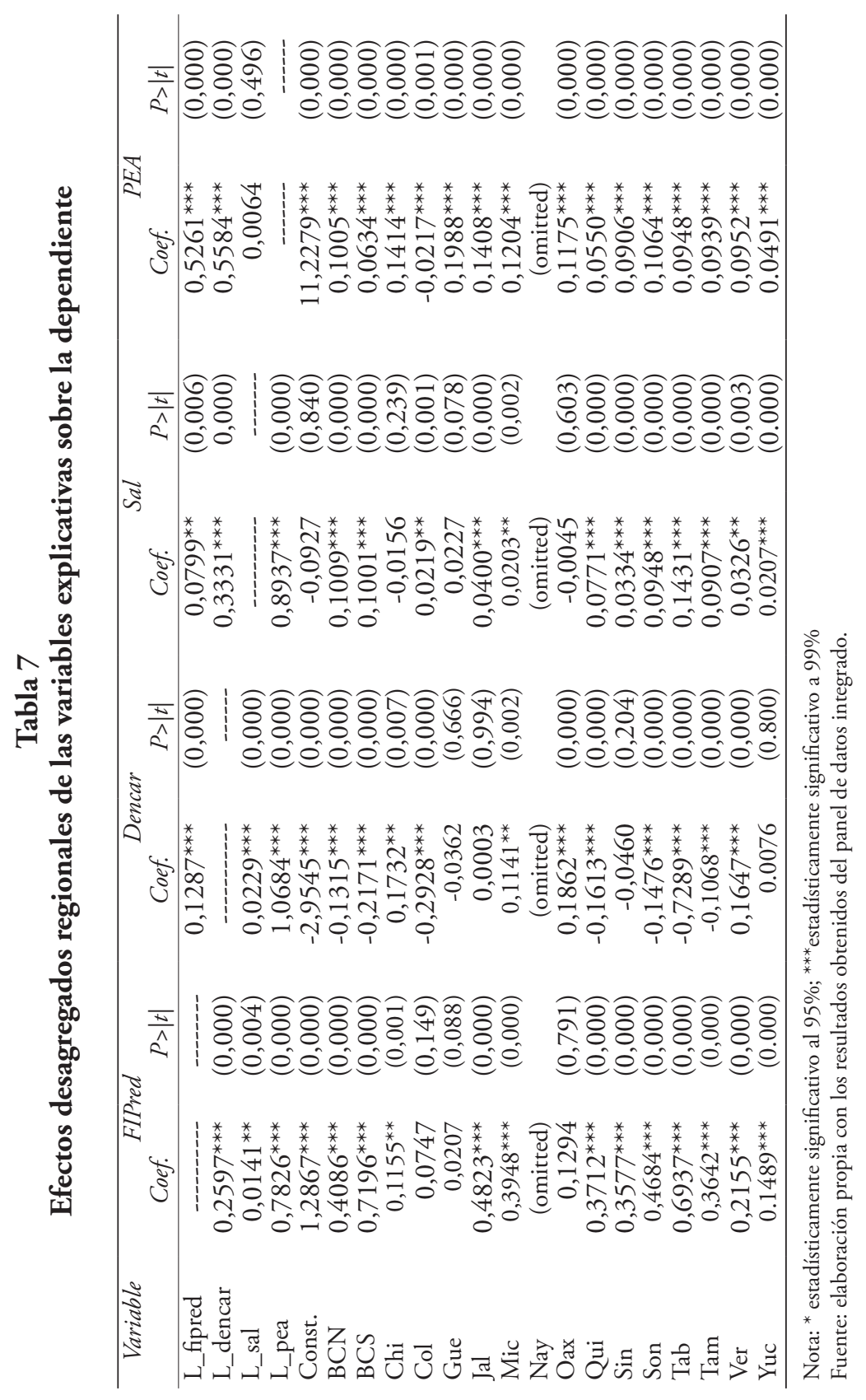


Tabla 8

Datos y resultados de la regresión no lineal entre el Factor de Infraestructura Portuaria promedio de las regiones $\left(\right.$ FIPred $\left._{\text {PROM }}\right)$ y el Impacto del FIP sobre PIB (IMFP)

\begin{tabular}{lcrr}
\hline \multicolumn{4}{c}{ Impacto $(I M F P)=\varphi_{1} F_{\text {FROM }}{ }^{2}+\varphi_{2} F I P_{P R O M}$} \\
\hline \multicolumn{4}{c}{ Estadístico $T$} \\
\hline$\varphi_{1}$ & Coef. & $-3,2471$ & $P>|t|$ \\
$\varphi_{2}$ & $-0,3998^{* *}$ & 5,0937 & $(0,0064)$ \\
$\mathrm{R}^{2}$ & $0,8534^{* * *}$ & & $0,0002)$ \\
$\mathrm{R}^{2}$ ajustada & & 0,1360 \\
\hline
\end{tabular}

Nota: ${ }^{* *}$ estadísticamente significativo a $95 \%$; ${ }^{* * *}$ estadísticamente significativo a $99 \%$

Fuente: elaboración propia con resultados obtenidos empleando el software Eviews 7 y Stata versión 13.

\section{Grafica 1}

\section{Regresión lineal entre el FIP y el Impacto del FIP en el PIB}

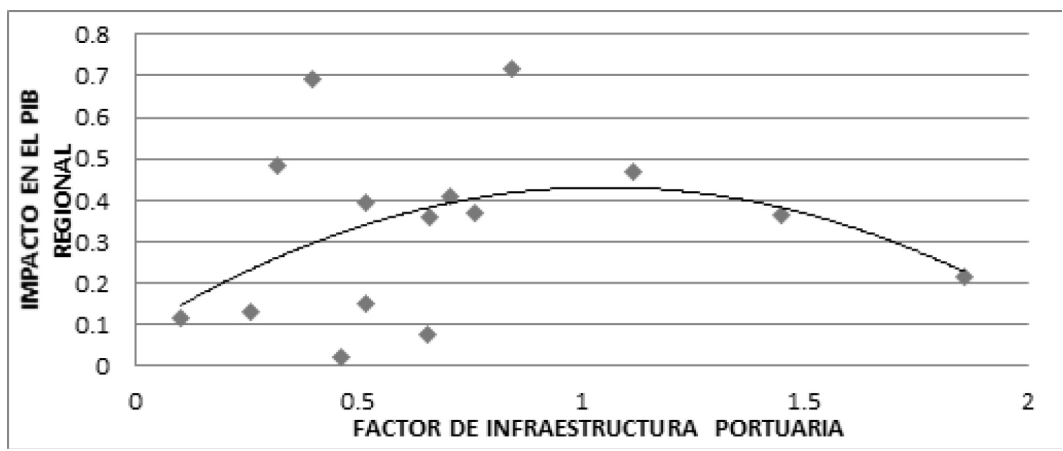

Fuente: elaboración propia a partir de los resultados del análisis del panel de datos.

lería con servicios de alimentos y bebidas y los Servicios inmobiliarios y alquileres representaron $26 \%$ del PIB.

\subsection{Efectos de la infraestructura portuaria por regiones}

Los efectos desagregados de cada una de las variables explicativas sobre la dependiente en el plano regional se colocaron en la tabla 7 y en su mayoría resultan altamente significativos y positivos.

$\mathrm{Al}$ analizar los desagregados del FIPred, todos son positivos, y las variables no desagregadas mantienen sus coeficientes positivos y altamente significativos, lo que nos permite afirmar que la infraestructura portuaria influye en el crecimiento económico en el plano regional. Los coeficientes 
de la regresora FIPred, resultan con menor valor para los estados de Guerrero, Colima, Oaxaca y Chiapas, y carecen de significancia estadística para los tres primeros. Las regiones para las cuales un incremento marginal de la infraestructura portuaria tiene su mayor influencia sobre el PIB económico de la región resultaron BCS $(0,71)$, Tabasco $(0,69)$ y Jalisco $(0,48)$.

Los puertos más importantes de México por su volumen de carga tienen influencia positiva y significativa en el desarrollo regional, con impactos marginales de 0,39 para la región de Michoacán (puerto de Lázaro Cárdenas), 0,36 para la región de Tamaulipas (puerto de Altamira), 0,21 para la región de Veracruz, (puerto de Veracruz y Coatzacoalcos). El efecto desagregado del FIB en el PIB de la región de Colima no tiene significancia estadística; este resultado hace difícil afirmar algo sobre el crecimiento económico regional del estado vinculada a los cambios en infraestructura que el puerto de Manzanillo ha tenido a lo largo del periodo de estudio. No obstante, en la última década Colima ha tenido un aumento en el PIB de $66 \%$, cuya composición ha variado reduciendo la participación del sector primario y secundario en $6.4 \%$ que ha pasado al sector terciario, particularmente al sector comercio y al sector transportes quienes participaron en 2013 con el $17.0 \%$ y $11.9 \%$ del PIB regional, respectivamente.

Al desagregar la densidad carretera (Dencar), los coeficientes tienen un comportamiento poco claro ya que presentan influencias significativas tanto positivas como negativas, y algunos casos no significativos. Colima, Tabasco y Jalisco son los estados con mayor densidad carretera, sin embargo esta característica parece no influir en su crecimiento regional, ya que presentan coeficientes negativos en este análisis, mientras que las regiones donde los incrementos marginales en densidad carretera tienen mayor influencia positiva en el incremento del PIB son Chiapas, Oaxaca y Michoacán, los cuales son tres de los cuatro estados con menor PIB per cápita del país, esto implica que en los estados más pobres los incrementos en infraestructura brindan mayores beneficios marginales.

En general el número de vuelos $(S A L)$ considerado como una variable proxy de la interconectividad internacional, tiene poca influencia con el $P I B$ regional, encontrándose valores opuestos, de baja significancia y de baja contribución, particularmente se observa que los coeficientes no significativos corresponden a los estados de Chiapas, Guerrero y Oaxaca. El mayor impacto de las salidas internacionales aéreas en el PIB, se observa para las regiones Baja California Sur y Quintana Roo, las cuales son regiones turísticas cuyo aforo es mayoritariamente internacional y el estado de Tabasco que tiene participación en la actividad petrolera. No obstante, los resultados encontrados con esta variable no son contundentes. 
Se puede observar que todos los coeficientes desagregados de la variable $P E A$ son altamente significativos. La PEA tiene influencia positiva, con un valor que va de un 0,04 a 0,19, sobre el PIB regional. Quintana Roo y Baja California Sur presentan la influencia más pequeña y junto con Colima que presenta una relación negativa, son estados en donde el saldo migratorio es positivo con incremento en la población desocupada (Inegi, 2014). Los coeficientes más altos corresponden a Guerrero, Chiapas, Jalisco, Michoacán y Oaxaca donde el saldo migratorio es negativo.

\section{Conclusiones}

La disponibilidad de infraestructura portuaria tiene una influencia positiva en el crecimiento económico regional. La influencia de la infraestructura portuaria no sólo depende de su disponibilidad, sino que es afectada por el uso y aprovechamiento, el cual es determinado por la demanda de servicios de transporte. Por ello, la dotación de infraestructura portuaria debe estar vinculada con proyectos y políticas públicas que fomenten la explotación, el uso económico y el beneficio social de las infraestructuras. Las regiones que presentan el menor Factor de infraestructura portuaria al mismo tiempo figuran entre las de PIB más bajo: Chiapas, Oaxaca y Guerrero. Diversas regiones de litoral mexicano cuentan con infraestructura portuaria menor cuyo incremento marginal podría brindar elementos de accesibilidad y conectividad lo que permitiría determinar nuevos patrones de movilidad, flujos comerciales, así como localizaciones industriales y residenciales con impacto económico regional mayor. Al mismo tiempo la inversión marginal concentrada en algunos grandes puertos no resulta proporcional a los impactos generados en el crecimiento, lo que agrava la disparidad regional. Este resultado es consistente con estudios similares (IFMO, 2007; Deng et al., 2014).

Con el desarrollo del intermodalismo y la mejora operativa, la región espacial de influencia de los grandes puertos se amplió, las regiones portuarias originales se rompieron a favor de un hinterland común competido por varios puertos simultáneamente en la medida en que los modos de transporte lo permiten: Jalisco uno de los estados con el FIP más bajo se sirve del hinterland portuario del estado de Colima, por su parte, Veracruz, quien posee el FIP más grande, compite con Colima y Michoacán, por brindar servicios a las regiones centrales e incorporarlas a su hinterland.

En cuanto a la diferencia geográfica o el tipo de tráfico entre los puertos del Pacífico y del Golfo de México y Mar Caribe y alguna posible relación entre ésta y su crecimiento económico, se realizaron análisis econométricos con los tres modelos sin encontrar resultados consistentes y significativos que puedan sugerir una relación o merezcan su mención. 
No obstante, sería recomendable profundizar el estudio con otras metodologías. Al respecto, también resulta importante desagregar el presente estudio para obtener una visión más detallada y amplia de la influencia de los puertos no sólo en el crecimiento sino en las variables que describen el desarrollo regional cuyo entendimiento permita comprender más ampliamente los beneficios regionales y que permita orientar las decisiones de inversión en infraestructura con un enfoque social.

Por otro lado, una ampliación en el periodo de análisis de la presente investigación anterior a 1996 puede brindar elementos para una comprensión más efectiva que valore los resultados brindados por la privatización portuaria.

\section{Fuentes consultadas}

Ahmed, Vaqar, Abbas Ahsan y Saira Ahmed (2013), "Public infrastructure and economic growth in Pakistan: a Dynamic CGE-Microsimulation Analysis", en John Cockburn, Yazid Dissou, Jean Yves Duclos y Luca Tiberti (eds.), Infrastructure and Economic Growth in Asia, Springer Cham Heidelberg, New York, Estados Unidos de América, pp. 117-143.

Aschauer, David (1998), "The role of public infraestructure capital in mexican economic growth", Economía Mexicana, Nueva Epoca, VII (1), Centro de Investigación y Docencia Económicas, Ciudad de México, México, pp. 47-78.

Aschauer, David (1989), "Is public expenditure productive?" Journal of Monetary Economics, 23 (2), Elsevier, Amsterdam, Holanda, pp. 177-200.

Barro, Robert (1988), "Government Spending in a simple model of endogenous growth", National Bureau of Economic Research, Working Papers núm. 2588, National Bureau of Economic Research, Massachusetts, Estados Unidos de América, pp. 103-125.

Berechman, Joseph, Ozmen, Dilruba, y Ozbay, Kaan (2006), “Empirical analysis of transportation investment and economic development at state, county and municipality levels", Transportation, 33 (6), Springer Science+Business Media, New York, Estados Unidos de América, pp. 537-551.

Cantos, Pedro, Mercedes Gumbau-Albert y Joaquín Maudos (2005), "Transport infrastructures, spillover effects and regional growth: 
evidence of the Spanish case", Transport Reviews, 25 (1), Taylor \& Francis, Abingdon, Reino Unido, pp. 25-50.

Carrillo-Murillo, David (2011), Demand and supply interactions in transport models: the case of hinterland transportation, Karlsruhe Papers in Economic Policy Research (Book 31), Nomos Publishers, Berlín, Alemania.

Chandra, Amitabh y Eric Thompson (2000), "Does public infrastructure affect economic activity? Evidence from the rural interstate highway system", Regional Science and Urban Economics, 30 (4), Elsevier, Amsterdam, Holanda, pp. 457- 490.

Deichmann, Uwe, Marianne Fay, Jun Koo y Somik Lall (2004), "Economic structure, productivity, and infrastructure quality in Southern Mexico", The Annals of Regional Science, 38 (3), Springer, New York, Estados Unidos de América, pp. 361-385.

Démurger, Sylvie (2001), "Infrastructure development and economic growth: an explanation for regional disparities in China?", Journal of Comparative Economics, 29 (1), Elsevier, Amsterdam, Holanda, pp. 95-117.

Deng, Taotao, Shuai Shao, Lili Yang y Xueliang Zhang (2014), "Has the transport-led economic growth effect reached a peak in China? A panel threshold regression approach", Transportation, 41 (3), Springer Science+Business Media, New York, Estados Unidos de América, pp. 567-587.

Ducruet, César (2009), "Port regions and globalization", en Theo Notteboom y César Ducruet, Peter de Langen, (eds.), Ports in Proximity: Competition and Coordination among Adjacent Seaports, Ashgate, Burlington, Estados Unidos de América, pp. 41-53.

Evans, Paul y Georgios Karras (1994), "Is government capital productive? Evidence from a panel of seven countries", Journal of Macroeconomics, 16 (2), Elsevier, Amsterdam, Holanda, pp. 271-279.

Fujita, Masahisa, Paul Krugman y Anthony Venables (2001), The spatial economy: Cities, regions and international trade, MIT Press, Massachusetts, Estados Unidos de América. 
Garcia-Milà, Teresa, Therese McGuire y Robert Porter (1996), “The effect of public capital in state-level production functions reconsidered", Reviews on Economics Statistics, 78 (1), Elsevier, Boston, Estados Unidos de América, pp. 177-180.

Garcia-Milà, Teresa y Therese McGuire (1992), "The contribution of publicly provided inputs to states' economies", Regional Science and Urban Economics, 22 (2), Elsevier, Amsterdam, Holanda, pp. 229-241.

Hong, Junjie, Zhaofang Chu y Qiang Wang (2011), "Transport infrastructure and regional economic growth: evidence from China", Transportation, 38 (5), Springer, New York, Estados Unidos de América, pp. 737-752.

IFMO (Institut für Mobilitätsforschung) (2007), Transport, Trade and Economic Growth. Coupled or Decoupled?, Springer Berlín Heidelberg, Berlín, Alemania.

Inegi (Instituto Nacional de Estadística y Geografía) (2014), Anuario estadístico y geográfico de los Estados Unidos Mexicanos 2014, Instituto Nacional de Estadística y Geografía, Aguascalientes, México.

Inegi (Instituto Nacional de Estadística y Geografía) (2013) Anuario estadístico de los Estados Unidos Mexicanos 2013, Instituto Nacional de Estadística Geografía e Informática, Aguascalientes, México.

Inegi (Instituto Nacional de Estadística Geografía e Informática) (2010), Regiones Socioeconómicas de México 2010, Instituto Nacional de Estadística Geografía e Informática, Aguascalientes, México.

Johansson, Börje (1993), "Infrastructure, accessibility and economic growth", International Journal of Transport Economics/Rivista internazionale di economia dei trasporti, 20 (2), Accademia Editoriale, Roma, Italia, pp. 131-156.

Lakshmanan, T. R., Peter Nijkamp, Piet Rietveld y Teodoor Verhoef (2001), "Benefits and costs of transport" Papers in Regional Science, 80 (2) Springer-Verlag, Berlín, Alemania, pp. 139-164. 
Lall, Somik (2007), "Infrastructure and regional growth, growth dynamics and policy relevance for India", The Annals of Regional Science, 41 (3), Springer Berlin Heidelberg, Berlín, Alemania, pp. 581-599.

Levin, Andrew, Lin Chien-Fu, Chu y Chia-Shang James (2002), "Unit root tests in panel data: asymptotic and finite-sample properties", Journal of Econometrics, 108 (1), Elsevier BV, North-Holland, Holanda, pp. 1-24.

Looney, Robert y Peter Frederiksen (1981), "The regional impact of infrastructure investment in México", Regional Studies, 15 (4), Routledge, London, Reino Unido, pp. 285-296.

Moomaw, Ronald y Martin Williams (1991), “Total factor productivity growth in manufacturing: further evidence from the States", Journal of Regional Science, 31 (1), Wiley-Blackwell, Pennsylvania, Estados Unidos de América, pp. 17-34.

Mody, Ashoka y Wang Fang-Yi (1997), "Explaining industrial growth in coastal China: economic reform and what else?", World Bank Economic Review, 11 (2), Oxford University Press, Oxford, Reino Unido, pp. 293-325.

Ozbay, Kaan, Dilruba Ozmen-Ertekiny Joseph Berechman (2007), “Contribution of transportation investments to county output", Transport Policy, 14 (4), Elsevier, England, Reino Unido, pp. 17-329.

Paaswell, Robert (1997), "Why Panels for Transportation Planning?" en Golob, Thomas; Kitamura, Ryuichi y Long, Lyn (eds.), Panels for Transportation Planning, Springer Science \& Business Media, New York, Estados Unidos de América, pp. 3-14.

Paredes, Víctor (2007), Privatización de Puertos en México: Reformas y Mercados de Servicios Portuarios, Centro de Investigación para el Desarrollo, Ciudad de México, México.

Raimond, Timothy y David Hensher (1997), "A Review of Empirical Studies and Applications", en Thomas Golob, Ryuichi Kitamura y Lyn Long (eds.), Panels for Transportation Planning, Springer Science \& Business Media, New York, Estados Unidos de América, pp. 15-72. 
Rietveld, Piet y Peter Nijkamp (1992), "Transport and regional development”, Research Memorandum, núm 50, Serie Research Memoranda, VU University Amsterdam, Amsterdam, Holanda, pp. $1-21$.

SCT (Secretaría de Comunicaciones y Transportes) (2015), "Catastro Portuario Nacional", Secretaría de Comunicaciones y Transportes, Ciudad de México, México, <http://www.sct.gob.mx/puertos-ymarina/puertos/catastro/>, 4 de enero de 2015.

SCT (Secretaría de Comunicaciones y Transportes) (2014), Prontuario del Servicio de Transporte Marítimo Regular entre México y el Mundo 2014, Secretaría de Comunicaciones y Transportes, Ciudad de México, México.

SCT (Secretaría de Comunicaciones y Transportes) (2013), Anuario estadístico del sector 2013, Secretaría de Comunicaciones y Transportes, Ciudad de México, México.

Singh, Prakash y N. R. Bhanumurthy (2014), "Infrastructure development and regional growth in India" en Ambar Ghosh y Asim Karmakar (eds.), Analytical Issues in Trade, Development and Finance, Springer, New Delhi, India, pp. 321-341.

Song, Lili y Marina van Geenhuizen (2014), "Port infrastructure investment and regional economic growth in China: Panel evidence in port regions and provinces", Transport Policy, 36, Elsevier, England, Reino Unido, pp. 173-183.

Wu, JunJie y Munisamy Gopinath (2008), "What causes spatial variations in economic development in the United States?", American Journal of Agricultural Economics, 90 (2), Oxford University Press, Oxford, Reino Unido, pp. 392-408.

Yoo, Seung-Hoon (2006), "Seaport infrastructure investment and economic growth in Korea", International Journal of Critical Infrastructure, 2 (1), Elsevier, Amsterdam, Holanda, pp. 1-9.

Zhang, Xueliang (2008), "Transport infrastructure, spatial spillover and economic growth: Evidence from China", Frontiers of Economics in China, 3 (4), Springer Publishing, Berlín, Alemania, pp. 585-597. 
Recibido: 16 de enero de 2016.

Corregido: 1 de julio de 2016. Aceptado: 7 de marzo de 2017.

Isidro Enrique Zepeda-Ortega. Candidato a grado de doctor en Ciencias Económicas por el Instituto Politécnico Nacional. Maestro en Ingeniería campo Sistemas de Transporte por la Universidad Nacional Autónoma de México. Su línea de investigación actual es Economía de los transportes y desarrollo económico regional. Publicaciones: "Análisis logístico en la industria de construcción de vivienda en serie: un estudio de caso en México" en Sandra Téllez, Miguel Cedillo y José Alfedo Jiménez (coords.), Logística y cadena de suministros: retos y desafíos en México, Universidad Politécnica de Guanajuato, Cortazar, México, pp. 80-101 (2015).

Gerardo Ángeles-Castro. Doctor en economía por la Universidad de Kent, Inglaterra; maestro en economía política internacional por la Universidad de Warwick, Inglaterra. Actualmente es profesor-investigador en la Sección de Estudios de Posgrado e Investigación, Escuela Superior de Economía, y coordinador de la Red de Desarrollo Económico del Instituto Politécnico Nacional. Es miembro del Sistema Nacional de Investigadores, nivel II. Sus líneas de investigación son desarrollo económico y econometría aplicada. Algunas de sus publicaciones: "Determinants of leverage in mining companies, empirical evidence for Latin American countries", Contaduría y Administración, 6 (1), Universidad Nacional Autónoma de México, Ciudad de México, México, pp. 26-40 (2016); en coautoría: "Crecimiento económico y desarrollo humano en la ciudad de México con respecto a un entorno nacional: una perspectiva neoclásica y dualista", Economía, Sociedad y Territorio 13 (42), El Colegio Mexiquense A. C., Toluca, México, pp. 431-457 (2013); "Tendencia y perspectiva de la economía global y su impacto en la economía mexicana”, Argumentos, 25 (70), Universidad Autónoma Metropolitana unidad Xochimilco, Ciudad de México, México, pp. 129-148 (2012); "Economic Liberalisation and income distribution, theory and evidence in Mexico", en Gerardo Ángeles, Ignacio Perrotini y Humberto Ríos (eds.), Market liberalism, growth and economic development in Latin America; Routledge, Oxon, Reino Unido, pp. 195-219 (2011); "La relación de la inversión extranjera directa con el crecimiento y la desigualdad del ingreso: Un análisis regional para México”, Ecorfan Journal, 1 (1), Ecorfan, Ciudad de México, México, pp. 10-33 (2010); "The effects of economic liberalization on income distribution: a panel data analysis", Wages, employment, distribution and growth, Palgrave Macmillan, Basingstoke, Reino Unido, pp. 151-180 (2006). 
David Guillermo Carrillo-Murillo. Doctor en economía e ingeniería de negocios por el Karlsruhe Institut für Technologie (KIT), Alemania con mención Summa Cum Laude; maestro en economía de los transportes por la Universidad de Lumière Lyon II, Francia. Actualmente es coordinador de Proyectos de Transporte y Movilidad en la empresa DTP Consultores. Participó como director de corredores multimodales y logística, en la Dirección General de Transporte Ferroviario y Multimodal de la Secretaría de Comunicaciones y Transportes donde coordinó técnicamente el Sector de Logística Nacional y la vinculación con los países de Centroamérica y Norteamérica, el Programa de Apoyo Federal al Transporte Masivo en conjunto con Banobras, el Anuario estadístico de Terminales de Carga y participó en la planeación de diversos proyectos del sector ferroviario de carga y pasajeros, proyectos multimodales, extensiones de líneas de metros, proyectos de Bus Rapid Transit y la Conectividad del Nuevo Aeropuerto Internacional de la Ciudad de México. Como consultor, ha participado en diversos proyectos en los sectores portuario, carretero, marítimo, ferroviario, multimodal, redes de comunicaciones y transporte urbano. Ha sido profesor en la Universidad La Salle, campus Ciudad de México y asistente de profesor en la Università degli Studi di Genova, en Italia. Su línea de investigación actual es transporte y logística así como economía de los transportes. Entre sus últimas publicaciones destacan: "Refrigerated container versus bulk: evidence from the banana cold chain", Maritime Policy \& Management, 42 (3), Taylor \& Francis, Routledge, London, Reino Unido, pp. 228-245 (2015); “A model for the formation of colloidal structures in freight transportation: The case of hinterland terminals", Transportation Research Part E, 49 (1), Elsevier, Amsterdam, Holanda, pp. 55-70 (2013); "Assessment of policy strategies to develop intermodal services: The case of inland terminals in Germany", Transport Policy, núm. 24, Elsevier, England, Reino Unido, pp. 168-178 (2012); "Stochastic distribution of intermodal transport freight flows in hinterlands", International Journal of Transport Economics, núm. 38, Accademia Editoriale, Roma, Italia, pp. 9-28 (2011); Demand and supply interactions in transport models: the case of hinterland transportation, Karlsruhe Papers in Economic Policy Research (Book 31), Nomos Publishers, Berlín, Alemania (2010). 